\title{
Effect of the Jianpi Bushen Prescription on expressions of the Wnt3a and Cyclin D1 genes in radiation-damaged mice
}

\author{
D.C. He*, J.J. Xiao*, Y. Zhang, H. Lin, X.J. Ding and Y. Tu \\ Wuhan General Hospital of Guangzhou Military Region, Wuhan, \\ Hubei, China \\ *These authors contributed equally to this study. \\ Corresponding author: D.C. He \\ E-mail: xiaojingjing5961@126.com
}

Genet. Mol. Res. 12 (4): 4137-4146 (2013)

Received February 19, 2013

Accepted August 27, 2013

Published October 3, 2013

DOI http://dx.doi.org/10.4238/2013.October.3.1

\begin{abstract}
The effects of the traditional Chinese drug Jianpi Bushen Prescription (JBP) were investigated on expressions of Wnt $3 a$ and Cyclin D1 genes in radiation-damaged mice. The radiation damage model was induced in Kumming mice by single total body irradiation treatment for 9 days. Mice were divided into the radiation group, lowdose (100\%) JBP group, high-dose (200\%) JBP group, or batyl alcohol group (positive control), which were administered twice a day for 9 days. mRNA and protein expressions of Wnt $3 a$ were detected in bone marrow mononuclear cells by real-time polymerase chain reaction and Western blot, whereas Cyclin D1 mRNA was detected by in situ hybridization. $W n t 3 a$ expressions were significantly downregulated in the radiation damage model group compared with all other groups $(\mathrm{P}<0.05)$. The positive cell rate of Cyclin D1 mRNA expression and the number of granulocyte macrophage colonies were significantly decreased in the radiation damage model group relative to all other groups $(\mathrm{P}<0.05)$. Furthermore, mRNA and protein expressions of Wnt $3 a$, the positive cell rate of Cyclin D1 mRNA expression in bone marrow cells, and the number of granulocyte macrophage colonies were all significantly higher in the low-dose JBP group than in the high-dose JBP group (P
\end{abstract}


$<0.05)$. In summary, JBP plays a protective role on radiation-induced bone marrow through the activation of the Wnt $3 a$ signaling pathway, and promotes the transcription and expression of Cyclin D1.

Key words: Jianpi Bushen Prescription; Radiation damage; Wnt $3 a$; Cyclin D1; Granulocyte macrophage colony generation; Animal model

\section{INTRODUCTION}

Radiation is an important risk factor of human health. Exposure to radiation can result in bone marrow hematopoietic disorders, decreased immune function due to the basic lesions of acute radiation sickness, and can even induce cell mutations, cancer, death, and other serious consequences (Kamiya and Sasatani, 2012; Wang et al., 2013). Exposure to radiation, and therefore radiation damage, is increasing along with increasing scientific and technological developments. The active ingredients of traditional Chinese medicine play an increasingly important role in radiation protection due to their demonstrated activities in improving hematopoietic function and enhancing immune function, thereby preventing or mitigating the harm of radiation for human health (Xu et al., 2012; Zhang et al., 2013). Jianpi Bushen Prescription (JBP; previously known as Digan oral liquid) has a protective function against radiation and chemical damage. Previous clinical trials indicated that JBP improves peripheral blood and enhances immunological function. Animal experiments have demonstrated further protective mechanisms of JBP against radiation damage with respect to marrow colony-stimulating factor, the expressions of apoptosis-related genes in marrow and spleen, and splenic histopathological changes. Recent studies have shown that changes in cell cycle-regulatory genes and the lack of control of transduction signaling pathways may be involved in the mechanism of radiation damage (Zhang et al., 2012a). The Wnt gene plays an important role in the regulation of the hematopoietic system and regulates hematopoietic cell proliferation by controlling the transcription of the $W n t 3 a$ protein, activating $\mathrm{Wnt} / \beta$-catenin signal transduction pathways, and decreasing the expression of downstream target genes. Therefore, in the present study, the effect of JBP on the expressions of Wnt $3 a$ and Cyclin D1, and the number of granulocyte macrophage colonies were investigated. Results of this study will provide further understanding related to the protection mechanism of Chinese medicines of the DGOL-formulae, which have known properties of fortifying the spleen, supplementing the kidney, reinforcing gas, and nourishing blood in response to radiation damage.

\section{MATERIAL AND METHODS}

\section{Drug preparation}

JBP was mainly composed of a large dose of prepared rehmannia root and honey-fried licorice root, supplemented with angelica, radix astragalus, and tangerine peel. JBP was prepared by the Department of Pharmaceutical Preparation in hospital, through water decoction, filtration, and concentration adjustment. Doses of 100\% (1 g crude drugs $/ \mathrm{mL})$ and $200 \%(2 \mathrm{~g}$ crude drugs $/ \mathrm{mL}$ ) liquid medicine were prepared, subpackaged, and preserved. Batyl alcohol (BA) was obtained by GKH Pharmaceuticals Ltd. (batch No. 20100725). The fluorescein iso- 
thiocyanate (FITC) rat anti-mouse CD45 antibodies were obtained from the Pharmigen Corporation. FITC-labeled sheep anti-mouse IgG was obtained from the Shenzhen JM Co., Ltd.

\section{Main reagents and apparatus}

The erythrocyte splitting liquor was purchased from the BA Phil Wuhan Company. The DYCZ-40 Electrorotation Generator was manufactured by the Beijing LiuYi Instrument Factory. Trizol was purchased from the Invitrogen Company (USA). The reverse transcriptase and first-chain synthetic cDNA kits were purchased from the Fermentas Company. The mouse Cyclin D1 mRNA hybrid kit was purchased from the Boster Company. GM-SF, low melting point agarose, fetal bovine serum, glutamine, and polyvinylidene fluoride (PVDF) film was purchased from Millipore Acrylamide, and the phenylmethylsulfonyl fluoride was bought from Amresco. The enhanced chemiluminescence (ECL) substrate solution was from Thermo NC15079. Other chemical reagents used were derived from traditional Chinese medicine. The micropipette was purchased from Eppendorf (Germany). The electro-thermostatic blast oven was bought from the Shanghai Jing Hong Laboratory Instrument Co., Ltd. The Dongsheng Innovation Biotechnology polymerase chain reaction (PCR) instrument was used, and a Shanghai ShunYu Constant Scientific Instrument Co. spectrophotometer was used. Hitachi HV-720 television cameras and HPIAS-1000 image analysis software packages were obtained from the Tongji Medical College of Huazhong University.

\section{Animals and grouping}

Sixty male Kunming mice (18 to $22 \mathrm{~g}$ ) were obtained from the Hubei Province Experimental Animal Research Center. Twelve mice were randomly selected in the normal control group. The 48 mice were treated with single total body irradiation (STBI) (dose: 6.0 Gy, dose rate: $450 \mathrm{~Gy} / \mathrm{min}$ ) using the linear accelerator X line for 9 days to induce the radiation damage mouse model. Decreased appetites and reduced activity levels were used as the criteria for successful modeling of mice, and compared with the normal group, the white blood cell count was significantly reduced in radiation damage model mice (normal group: $12.61 \mathrm{x}$ $10^{9} / \mathrm{L}$, radiation-damaged mice: $7.34 \times 10^{9} / \mathrm{L}$ ). According to the random number table, 48 mice were divided into the model group, low-dose JBP group (100\% concentration), high-dose JBP group (200\% concentration), and BA group (positive control). Normal control and model group mice were administered lavage with $0.2 \mathrm{mg} / 10 \mathrm{~g}$ sodium chloride twice a day. The other three groups were respectively administered lavage with a $0.1 \mathrm{~g} / \mathrm{kg}$ suspension low-dose JBP, high-dose JBP, or $0.2 \mathrm{~mL} / 10 \mathrm{~g}$ BA twice a day. The mice were sacrificed on the ninth day, and the bilateral femur and tibia bone marrow were collected.

\section{Real-time PCR for detection of Wnt3a mRNA expression}

Total RNA was extracted from the bilateral femur and tibia bone marrow using Trizol reagent. Primers used for the reverse transcription reaction were designed based on GenBank sequences using the Primer 5.0 software for self-analysis. After an initial denaturation for 2 min at $94^{\circ} \mathrm{C}, 40$ cycles of PCR were run. Each cycle consisted of 2 min at $50^{\circ} \mathrm{C}, 10 \mathrm{~min}$ at $95^{\circ} \mathrm{C}$, and $30 \mathrm{~s}$ at $95^{\circ} \mathrm{C}$, with a final extension for $30 \mathrm{~s}$ at $60^{\circ} \mathrm{C}$. The $\beta$-actin gene was amplified 
at the same time as reference. Cycle threshold $(\mathrm{Ct})$ values were obtained using the ECO Realtime PCR system software v3.0, and relative differences in gene expression were calculated with the $2-\Delta \Delta \mathrm{Ct}$ method.

\section{Western blot for detection of $\boldsymbol{W n t} 3 a$ protein expression}

Tissue weights of bilateral femur and tibia bone marrow were calculated, and RIPA erythrocyte splitting liquor was added to the cell suspension, blended in a centrifuge tube at $4^{\circ} \mathrm{C}$ for $5 \mathrm{~min}$, and the supernatant was collected and stored at $-20^{\circ} \mathrm{C}$. To determine the protein concentration, the same quality of erythrocyte splitting liquor (volume $\mathrm{x}$ protein concentration) was used with the addition of the same volume $2 \mathrm{X}$ electrophoresis loading buffer. After boiling for $3 \mathrm{~min}$ in a water bath, samples were loaded for electrophoresis (20 $\mathrm{mA}$ concentrate glue, $35 \mathrm{~mA}$ discrete glue). The transmembrane (tank transfer systems) was immersed in the PVDF membrane in Tris-buffered saline and Tween 20 sealing fluid containing 5\% bovine serum albumin, and then shaken at room temperature for $2 \mathrm{~h}$. Sealing fluid was then used to dilute the corresponding primary antibodies, which were incubated overnight at $4^{\circ} \mathrm{C}$. Samples were then incubated in secondary horseradish peroxidase-labeled antibodies, and then shaken at room temperature for $2 \mathrm{~h}$. ECL substrate fluid was then added and the fluorescence band was observed using X-ray film squash, developed, and fixed. The film was scanned, and the Bandscan software was used to analyze the gray value. Relative gene expression was represented by the ratio of the gray value of the gene to the gray value of GAPDH.

\section{Measurement of Cyclin D1 mRNA expression by in situ hybridization}

The mRNA nucleic acid fragment was extracted from bone marrow nucleated cells by adding $3 \%$ pepsin drops, diluted by citric acid, to bone marrow, which was then cut into sections for digestion at $37^{\circ} \mathrm{C}$ for 30 to $120 \mathrm{~min}$. Then, $20-\mu \mathrm{L}$ hybridization solution was added to each section, and the hybridization reaction was conducted at a constant temperature $\left(40^{\circ}-42^{\circ} \mathrm{C}\right)$ overnight. Biotinylated mouse anti-digoxin was added drop-wise at $37^{\circ} \mathrm{C}$ for $60 \mathrm{~min}$ or at $20^{\circ} \mathrm{C}$ for $120 \mathrm{~min}$. Sections were washed four times with $0.5 \mathrm{M}$ PBS for 5 min each. One drop each chromogenic agent A, B, and C (DBA Color Development Kit) was mixed with $1 \mathrm{~mL}$ distilled water, blended, and the mixture was added to the specimen. The color development time was 20-30 min. Hitachi HV-720 television camera radiography was used to collect images and the HPIAS-1000 image analysis software package was used for data collection.

\section{Measurement of the number of granulocyte macrophage colonies by the in vitro semi-solid agar single culture method}

After preparation of the bone marrow mononuclear cell suspension, a training system was established including $20 \%$ fetal bovine serum, $50 \mathrm{ng} / \mathrm{mL}$ GM-CSF, $0.3 \%$ agar, 4 $\mathrm{mM}$ glutamine, and $2 \times 10^{5} / \mathrm{mL}$ cell suspension. The system was cultured at $37^{\circ} \mathrm{C}$ in a $5 \%$ $\mathrm{CO}_{2}$ saturated humidity environment. The colony was observed after 10-14 days of culture, in which more than 40 cells grouped into a collection. 


\section{Statistical analysis}

Data are reported as means $\pm \mathrm{SD}$. Differences among groups were compared by oneway analysis of variance (ANOVA) followed by the Scheffé post hoc comparison test. A value of $\mathrm{P} \leq 0.05$ was accepted as significant.

\section{RESULTS}

\section{Effect of radiation damage (JBP) on Wnt3a expression in radiation-damaged mice}

Mice were treated with STBI (dose: $6.0 \mathrm{~Gy}$, dose rate: $450 \mathrm{~Gy} / \mathrm{min}$ ) using the linear accelerator $\mathrm{X}$ line for 9 days to induce radiation damage. The mice were administered lavage with $0.1 \mathrm{~g} / \mathrm{kg}$ suspension low-dose JBP, high-dose JBP, or BA for 9 days. The bilateral femur and tibia bone marrow were collected and the mRNA and protein expressions of Wnt $3 a$ in bone marrow cells were measured by real-time PCR and Western blot. As shown in Figures 1 and 2, compared with the control group, the mRNA and protein expressions of $W n t 3 a$ were significantly downregulated in the radiation damage model group (all $\mathrm{P}<0.05$ ). The low-dose JBP, high-dose JBP, and BA significantly upregulated the mRNA and protein expressions of $W n t 3 a$ compared to the radiation damage model group (all $\mathrm{P}<0.05$ ). However, the mRNA and protein expressions of $W n t 3 a$ were significantly higher in the low-dose JBP group than in the high-dose JBP group (all $\mathrm{P}<0.05$ ).

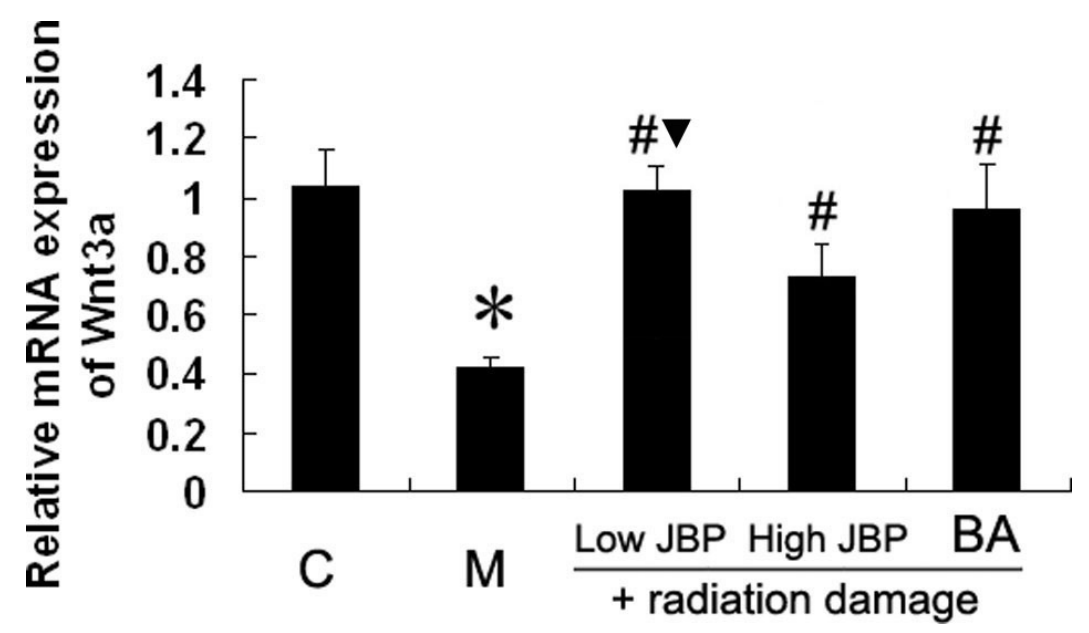

Figure 1. Effect of radiation damage and radiation of the Chinese drug Jianpi Bushen Prescription (JBP) on mRNA expression of Wnt $3 a$ in radiation-damaged mice. Mice were treated with single total body irradiation (dose: $6.0 \mathrm{~Gy}$, dose rate: $450 \mathrm{~Gy} / \mathrm{min}$ ) by using linear accelerator $\mathrm{X}$ line for 9 days to induce radiation damage mouse model. Mice were administered lavage with $0.1 \mathrm{~g} / \mathrm{kg}$ suspension low-dose JBP (100\%), high-dose JBP (200\%), and $0.2 \mathrm{~mL} / 10 \mathrm{~g}$ batyl alcohol (BA, positive control), twice a day for 9 days. The bilateral femur and tibia bone marrow were collected and the level of the Wnt $3 a$ mRNA in bone marrow cells was tested by real-time PCR. C = control group; $\mathrm{M}=$ radiation damage model group; Low JBP = low-dose JBP (100\%) group; High JBP $=$ high-dose JBP $(200 \%)$ group; BA = batyl alcohol group. Data are reported as means $\pm \mathrm{SD}(\mathrm{N}=12) . * \mathrm{P}<0.05$, compared to control group; ${ }^{*} \mathrm{P}<0.05$, compared to radiation damage model group; ${ }^{\mathbf{}} \mathrm{P}<0.05$, compared to highdose JBP + radiation damage group. 

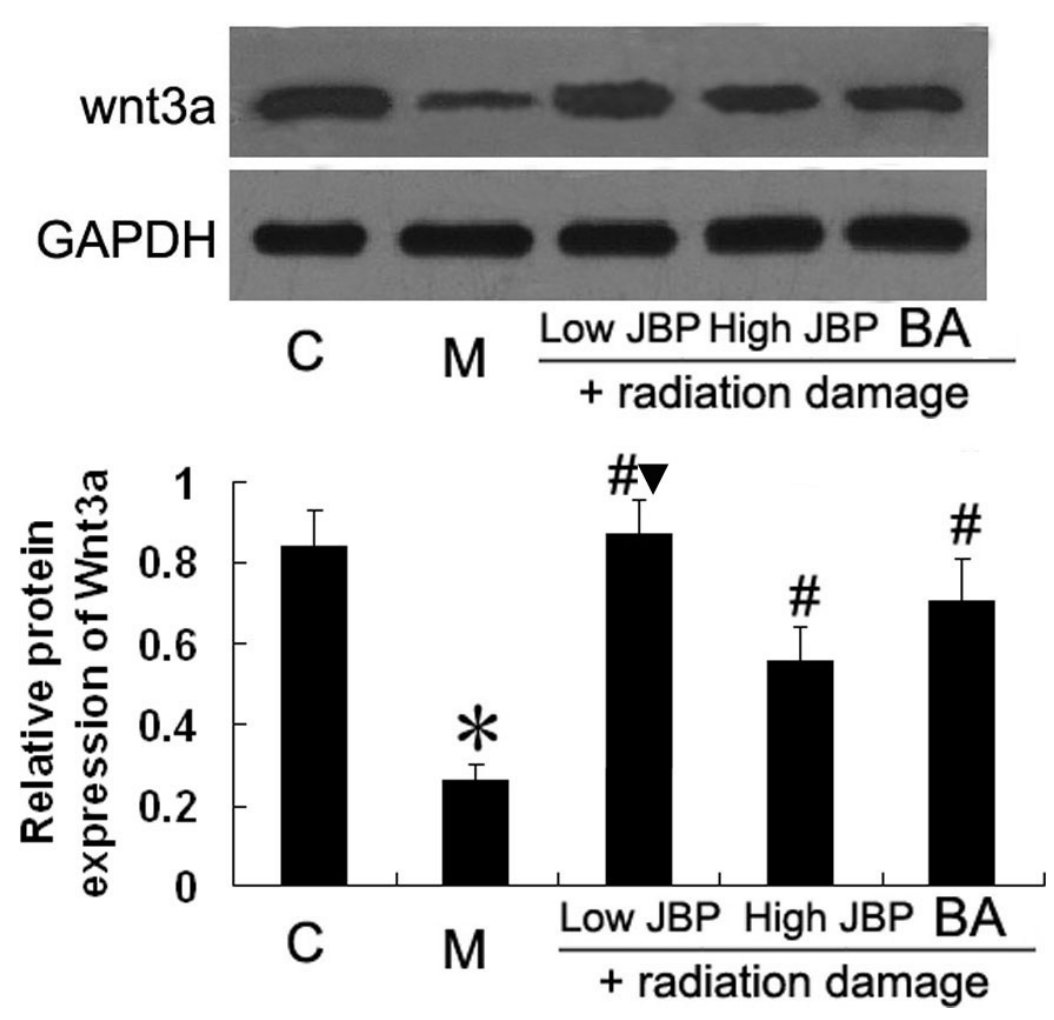

Figure 2. Effect of radiation damage and radiation of the Chinese drug Jianpi Bushen Prescription (JBP) on protein expression of $W n t 3 a$ in radiation-damaged mice. Mice were treated with single total body irradiation (dose: 6.0 Gy, dose rate: $450 \mathrm{~Gy} / \mathrm{min}$ ) by using linear accelerator $\mathrm{X}$ line for 9 days to induce radiation damage mouse model. Mice were administered lavage with $0.1 \mathrm{~g} / \mathrm{kg}$ suspension low-dose JBP (100\%), high-dose JBP (200\%), and 0.2 $\mathrm{mL} / 10 \mathrm{~g}$ batyl alcohol (BA, positive control), twice a day for 9 days. The bilateral femur and tibia bone marrow were collected and the level of the $W n t 3 a$ protein in bone marrow cells was tested by Western blot. $\mathrm{C}=$ control group; $\mathrm{M}=$ radiation damage model group; Low JBP = low-dose JBP $(100 \%)$ group; High JBP = high-dose JBP $(200 \%)$ group; $\mathrm{BA}=$ batyl alcohol group. Data are reported as means $\pm \mathrm{SD}(\mathrm{N}=12) . * \mathrm{P}<0.05$, compared to control group; ${ }^{*} \mathrm{P}<0.05$, compared to radiation damage model group; $\mathbf{} \mathrm{P}<0.05$, compared to high-dose JBP + radiation damage group.

\section{Effect of radiation damage and JBP on mRNA expression of Cyclin D1 in radiation-damaged mice}

Mice were treated with STBI (dose: $6.0 \mathrm{~Gy}$, dose rate: $450 \mathrm{~Gy} / \mathrm{min}$ ) using the linear accelerator $\mathrm{X}$ line for 9 days to induce radiation damage. Mice were administered lavage with $0.1 \mathrm{~g} / \mathrm{kg}$ suspension low-dose JBP, high-dose JBP, or BA for 9 days. The bilateral femur and tibia bone marrow were collected and the positive cell rate of Cyclin D1 mRNA expression in bone marrow cells was evaluated with in situ hybridization. As shown in Figure 3, compared with the control group, the positive cell rate of Cyclin D1 mRNA expression in bone marrow cells was significantly decreased in the radiation damage model group (all $\mathrm{P}<0.05$ ). The lowdose JBP, high-dose JBP, and BA all significantly increased the positive cell rate of Cyclin 
D1 mRNA expression in bone marrow cells compared to that of the radiation damage model group (all $\mathrm{P}<0.05$ ). The positive cell rate of Cyclin D1 mRNA expression in bone marrow cells was significantly higher in the low-dose JBP group than in the high-dose JBP group (P $<0.05)$.

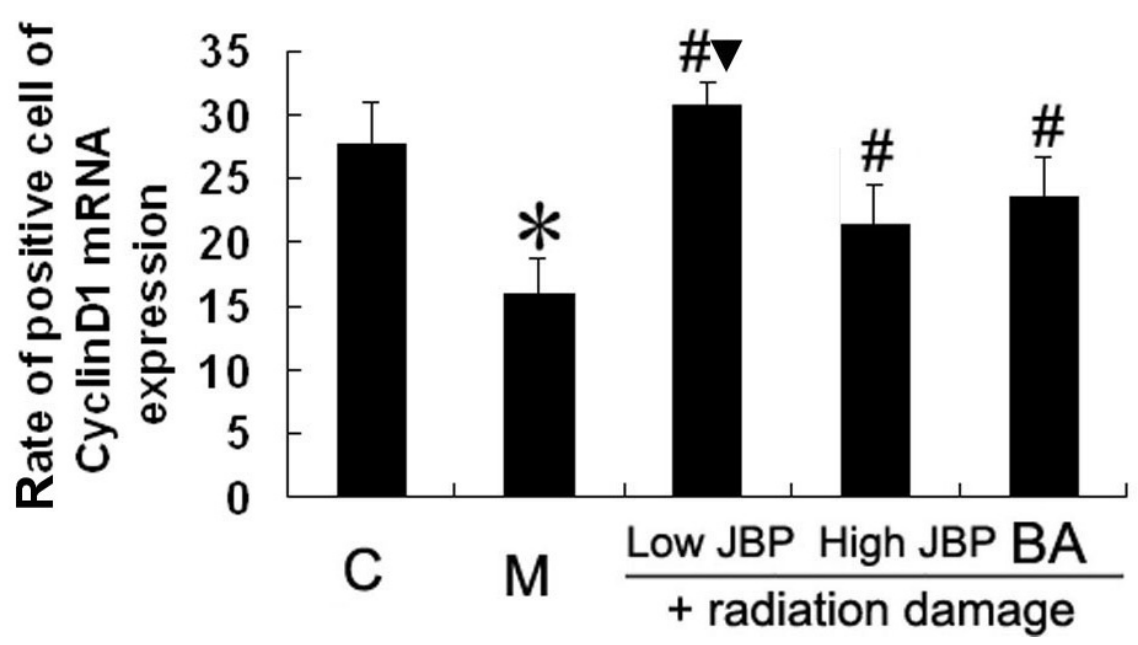

Figure 3. Positive cell rate of Cyclin D1 mRNA expression in murine bone marrow nucleated cells. Mice were treated with single total body irradiation (dose: $6.0 \mathrm{~Gy}$, dose rate: $450 \mathrm{~Gy} / \mathrm{min}$ ) by using linear accelerator $\mathrm{X}$ line for 9 days to induce radiation damage mouse model. Mice were administered lavage with $0.1 \mathrm{~g} / \mathrm{kg}$ suspension low-dose JBP (100\%), high-dose JBP (200\%), and $0.2 \mathrm{~mL} / 10 \mathrm{~g}$ batyl alcohol (BA, positive control), twice a day for 9 days. The bilateral femur and tibia bone marrow were collected. Positive cell rate of Cyclin D1 mRNA expression in bone marrow cells was tested by in situ hybridization. $\mathrm{C}=$ control group; $\mathrm{M}=$ radiation damage model group; Low $\mathrm{JBP}=$ low-dose JBP $(100 \%)$ group; High JBP = high-dose JBP (200\%) group; BA = batyl alcohol group. Data are reported as means $\pm \mathrm{SD}(\mathrm{N}=12)$. ${ }^{*} \mathrm{P}<0.05$, compared to control group; ${ }^{\mathrm{P}}<0.05$, compared to radiation damage model group; $\mathbf{} \mathrm{P}<0.05$, compared to high-dose JBP + radiation damage group.

\section{Effect of radiation damage and JBP on the number of granulocyte macrophage colonies in radiation-damaged mice}

Mice were treated with STBI (dose: $6.0 \mathrm{~Gy}$, dose rate: $450 \mathrm{~Gy} / \mathrm{min}$ ) using the linear accelerator $\mathrm{X}$ line for 9 days to induce radiation damage. Mice were administered lavage with $0.1 \mathrm{~g} / \mathrm{kg}$ suspension low-dose JBP, high-dose JBP, and BA for 9 days. The bilateral femur and tibia bone marrow were collected and the number of granulocyte macrophage colonies was evaluated by the in vitro agar single semisolid culture method. As shown in Figure 4, compared with the control group, the number of granulocyte macrophage colonies was significantly decreased in the radiation damage model group (all $\mathrm{P}<0.05$ ). Low-dose JBP, high-dose JBP, and BA all significantly increased the number of granulocyte macrophage colonies compared to the radiation damage model group (all $\mathrm{P}<0.05$ ). However, the number of granulocyte macrophage colonies was significantly higher in the low-dose JBP group than in the high-dose JBP group $(\mathrm{P}<0.05)$. 


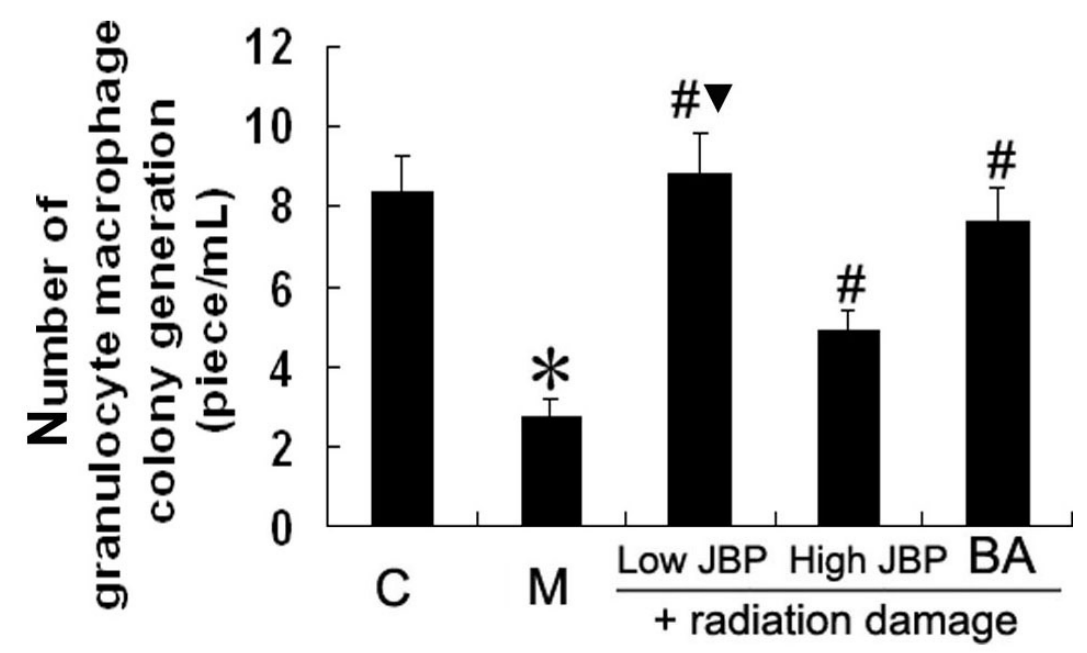

Figure 4. Effect of radiation damage and radiation of the Chinese drug Jianpi Bushen Prescription (JBP) on the number of granulocyte macrophage colony generation in radiation-damaged mice. Mice were treated with single total body irradiation (dose: $6.0 \mathrm{~Gy}$, dose rate: $450 \mathrm{~Gy} / \mathrm{min}$ ) by using linear accelerator $\mathrm{X}$ line for 9 days to induce radiation damage mice model. Mice were administered lavage with $0.1 \mathrm{~g} / \mathrm{kg}$ suspension low-dose JBP (100\%), high-dose JBP (200\%), and $0.2 \mathrm{~mL} / 10 \mathrm{~g}$ batyl alcohol (BA, positive control), twice a day for 9 days. The bilateral femur and tibia bone marrow were collected and the number of granulocyte macrophage colony generation was tested by in vitro single agar semisolid culture method. $\mathrm{C}=$ control group; $\mathrm{M}=$ radiation damage model group; Low $\mathrm{JBP}=$ low-dose JBP (100\%) group; High JBP = high-dose JBP (200\%) group; BA = batyl alcohol group. Data are reported as means $\pm \mathrm{SD}(\mathrm{N}=12)$. ${ }^{*} \mathrm{P}<0.05$, compared to control group; $\mathrm{P}<0.05$, compared to radiation damage model group; ${ }^{\mathrm{D}} \mathrm{P}<0.05$, compared to high-dose JBP + radiation damage group.

\section{DISCUSSION}

In traditional Chinese medicine, radiation damage belongs to the category of "deficiency of the spleen and kidney, lack of blood and gas". Therefore, the principle of the treatment is "to benefit gas and enrich blood". Traditional Chinese medicine considers the spleen as the source of gas and blood biochemistry, whereas the kidney governs bones, fresh marrow, and stores essential substances. Therefore, fortifying the spleen and supplementing the kidney are the most important methods to combat radiation damage. The JBP is an empirical formula that was developed in the Department of Traditional Medicine, Wuhan General Hospital. JBP is mainly composed of prepared rehmannia root and honey-fried licorice root, supplemented by angelica, radix astragalus, and tangerine peel, which achieves the effect of fortifying the spleen and supplementing the kidney to nourish the blood and marrow. A modern pharmacology study showed that rehmannia glutinosa has the effect of recovering the hematopoietic function of bone marrow suppression that is caused by radiation damage, and can also significantly promote the proliferation of hematopoietic stem cells and improve immune function (Ma et al., 2010). Angelica polysaccharide (AP) plays a significant role in promoting the proliferation and differentiation of liver hematopoietic cells and myeloid hematopoietic progenitor cells, particularly when peripheral blood cells are decreased and bone marrow is suppressed. AP could also improve the hematopoietic microenvironment to regulate hematopoietic cells (Liu 
et al., 2010; Hou et al., 2012). Radix astragalus has a comprehensive and sustained effect, including elevating whole blood cells, resulting in low levels of red blood cells, white blood cells, and platelets returning to normal levels in rats, promoting the secretion of hematopoietic cytokines, stimulating the function of the hematopoietic system, and improving the bone marrow suppression state caused by chemotherapy and radiotherapy (Gao et al., 2006; Zheng et al., 2011). In summary, the spleen and kidney plays a dual role in modern medicine to promote the regulation of hematopoietic cell proliferation and immune function.

Regulation of hematopoietic progenitor cells is the core function of the regulation of hematopoiesis. Hematopoietic progenitor cells are formed by the proliferation and differentiation of hematopoietic stem cells, and continue to develop into all types of mature blood cells under the control of a variety of hematopoietic growth factors by further proliferation and differentiation (Broxmeyer, 2011; Kollet et al., 2012).

Granulocyte macrophage hematopoietic progenitor cells are one type of hematopoietic progenitor cell. Granulocyte and monocyte macrophage cells originate from granulocyte macrophage hematopoietic progenitor cells, and the number of granulocyte macrophage colonies can influence the proliferative capacity of hematopoietic progenitor cells as well as immune function to some extent (Singh et al., 2012). Recent studies have shown that the Wnt signaling pathway, as an important regulator of hematopoietic stem/progenitor cells, plays an important role in the hematopoietic system (Staal et al., 2010; Ichii et al., 2012). Wnt proteins combine with surface receptor molecules of hematopoietic cells to complete the transfer of the Wnt signaling pathway through the phosphorylation and dephosphorylation of downstream proteins, ultimately acting on nuclear target genes, thus regulating the proliferation and differentiation of hematopoietic cells (Ming et al., 2012). The Wnt3a protein is an important activated protein in the $\mathrm{Wnt} / \beta$-catenin signaling pathway. $W n t 3 a$ proteins bind with a receptor, preventing the degradation of $\beta$-catenin. Therefore, $\beta$-catenin accumulates in the cytosol, and transfers to the nucleus once it reaches a certain level, which then initiates and activates the transcription of downstream target genes (such as c-myc, c-myb, Cyclin D1, ETS, and BMP4), so as to enhance the self-renewal capacity of hematopoietic stem cells (Kawaguchi-Ihara et al., 2008; Zhang et al., 2012b).

The normal cell cycle can be divided into the interphase and division periods, and the interphase can be classified into three stages, including the G1, the S, and the $\mathrm{G} 2$ phases. Cyclin D1 is one of the key factors controlling the conversion of the G1 to the S phase. Cyclin D1 controls the speed of cell proliferation by regulating the cell cycle (Dai et al., 2012). Cyclin D1 can also be used as a downstream target gene of the Wnt/b-catenin signal transduction pathway, and may initiate the pathway in some cases (Udhayakumar et al., 2007; Zhang et al., 2012a).

In the present study, the number of granulocyte macrophage colonies, the expression of $W n t 3 a$, and the positive cell rate of Cyclin D1 mRNA all significantly decreased in the radiation damage model groups compared with the control group. Low-dose JBP, high-dose JBP, and BA significantly increased the number of granulocyte macrophage colonies, the expression of $W n t 3 a$, and the positive cell rate of Cyclin D1 mRNA compared to the radiation damage model group, and the effect was much stronger in the low-dose group. These results indicate that JBP and BA intervention can promote transcription of the Wnt $/ \beta$-catenin signaling pathway, and activate the transcription and expression of Cyclin D1 in the pathway to promote the proliferation and maturation of hematopoietic stem/progenitor cells, thus inducing a protective effect on radiation-induced bone marrow. Moreover, in this experiment, the protection 
of radiation damage of the low-dose group was much better than that of the high-dose group, which indicates that high concentrations of the drug reduced the conduction of signaling pathways and transcription. This may be related with the large dose of prepared radix rehmanniae and the oiliness of licorice that can prevent gastrointestinal absorption; however, the specific mechanisms need further research.

In summary, results of this study demonstrated that JBP has a protective effect on radiation-induced bone marrow through the activation of the $W n t 3 a$ signaling pathway and promotion of the transcription and expression of Cyclin D1.

\section{REFERENCES}

Broxmeyer HE (2011). Insights into the biology of cord blood stem/progenitor cells. Cell Prolif. 44 (Suppl 1): 55-59.

Dai J, Zhang PH, Liu PS and Qu HH (2012). Expressions and significance of cyclinD1 in epithelial ovarian cancer cell 3AO. Zhonghua Yi Xue Za Zhi 92: 351-353.

Gao QT, Cheung JK, Li J, Chu GK, et al. (2006). A Chinese herbal decoction, Danggui Buxue Tang, prepared from Radix Astragali and Radix Angelicae Sinensis stimulates the immune responses. Planta Med. 72: 1227-1231.

Hou H, Bao Y, Li Q and Shi W (2012). Preparation of blood-deficient model and research of angelica polysaccharide on enriching blood in chickens. Evid. Based Complement. Alternat. Med. 2012: 965947.

Ichii M, Frank MB, Iozzo RV and Kincade PW (2012). The canonical Wnt pathway shapes niches supportive of hematopoietic stem/progenitor cells. Blood 119: 1683-1692.

Kamiya K and Sasatani M (2012). Effects of radiation exposure on human body. Nihon Rinsho 70: 367-374.

Kawaguchi-Ihara N, Murohashi I, Nara N and Tohda S (2008). Promotion of the self-renewal capacity of human acute leukemia cells by Wnt3A. Anticancer Res. 28: 2701-2704.

Kollet O, Canaani J, Kalinkovich A and Lapidot T (2012). Regulatory cross talks of bone cells, hematopoietic stem cells and the nervous system maintain hematopoiesis. Inflamm. Allergy Drug Targets 11: 170-180.

Liu C, Li J, Meng FY, Liang SX, et al. (2010). Polysaccharides from the root of Angelica sinensis promotes hematopoiesis and thrombopoiesis through the PI3K/AKT pathway. BMC Complement Altern. Med. 10: 79.

Ma J, Liang QD, Ma ZC, Wang YG, et al. (2010). Rehmanniae Radix provides most of the free fructose and glucose in Si-Wu-Tang decoction. Drug Discov. Ther. 4: 179-183.

Ming M, Wang S, Wu W, Senyuk V, et al. (2012). Activation of Wnt/beta-catenin protein signaling induces mitochondriamediated apoptosis in hematopoietic progenitor cells. J. Biol. Chem. 287: 22683-22690.

Singh P, Hu P, Hoggatt J, Moh A, et al. (2012). Expansion of bone marrow neutrophils following G-CSF administration in mice results in osteolineage cell apoptosis and mobilization of hematopoietic stem and progenitor cells. Leukemia 26: $2375-2383$.

Staal FJ and Luis TC (2010). Wnt signaling in hematopoiesis: crucial factors for self-renewal, proliferation, and cell fate decisions. J. Cell Biochem. 109: 844-849.

Udhayakumar G, Jayanthi V, Devaraj N and Devaraj H (2007). Interaction of MUC1 with beta-catenin modulates the Wnt target gene cyclinD1 in H. pylori-induced gastric cancer. Mol. Carcinog. 46: 807-817.

Wang C, Nakamura S, Oshima M, Mochizuki-Kashio M, et al. (2013). Compromised hematopoiesis and increased DNA damage following non-lethal ionizing radiation of a human hematopoietic system reconstituted in immunodeficient mice. Int. J. Radiat. Biol. 89: 132-137.

Xu P, Jia JQ, Jiang EJ, Kang LP, et al. (2012). Protective effect of an extract of Guipi Pill against radiation-induced damage in mice. Chin. J. Integr. Med. 18: 490-495.

Zhang H, Zhai Z, Wang Y, Zhang J, et al. (2013). Resveratrol ameliorates ionizing irradiation-induced long-term hematopoietic stem cell injury in mice. Free Radic. Biol. Med. 54: 40-50.

Zhang J, Gill AJ, Issacs JD, Atmore B, et al. (2012a). The Wnt/ $\beta$-catenin pathway drives increased cyclin D1 levels in lymph node metastasis in papillary thyroid cancer. Hum. Pathol. 43: 1044-1050.

Zhang W, Yao H, Wang S, Shi S, et al. (2012b). Wnt3a is involved in the early stage of miPSC and mESC haemopoietic differentiation. Cell Biol. Int. 36: 267-271.

Zheng KY, Choi RC, Cheung AW, Guo AJ, et al. (2011). Flavonoids from Radix Astragali induce the expression of erythropoietin in cultured cells: a signaling mediated via the accumulation of hypoxia-inducible factor-1alpha. $J$. Agric. Food Chem. 59: 1697-1704. 